\title{
Strong localization of the charge carriers in CdSe nanostructural films
}

\author{
Nader Ghobadi and Rostam Moradian
}

\begin{abstract}
Cadmium selenide nanoparticle films were deposited on usual glass substrate by chemical bath deposition method. Temperature dependence of dc conductivity of the present samples was studied. In the nanocrystalline materials due to small size of grains and confined charge carriers, the electronic states near the Fermi level are localized. When states are localized, the conduction occurs by hopping of carriers between the occupied and unoccupied localized states. Variable range hopping was identified as a predominant conduction mechanism and occurs above room temperature. The density of localized states near the Fermi level was observed to increase with increase of average grain size in the film.
\end{abstract}

Keywords: Nanoparticles; Chemical bath deposition; Density of localized states; Fermi level

\section{Background}

In disordered materials, such as amorphous semiconductor or some thin films, low temperature transport occurs between localized electronic states near the Fermi level $\left(E_{\mathrm{F}}\right)$. This transport depends on the density of states (DOS), location of Fermi level, and the temperature. Generally, at low temperatures (below about room temperature (RT)) and sometimes at temperatures above RT, the electrical conduction is interpreted by variable range hopping (VRH) mechanism [1-4]. High quality CdSe nanocrystals can be fabricated by several techniques such as electrodeposition, evaporation, and chemical bath deposition. In particular, chemical bath deposition (CBD) is one of the simplest and most economic techniques to grow stochiometrics CdSe thin films. A thin film formation takes place when ionic products exceed solubility. CBD method is presently attracting considerable attention as it does not require sophisticated instrumentation. It is relatively inexpensive, easy to handle, convenient for large area deposition, and capable of yielding good quality thin films. The characteristics of the chemically deposited CdSe thin films by CBD strongly depend on the growth condition; by changing the deposition key parameters, one can control thickness, size of the nanoparticles, and the energy band gap of the obtained thin films [5]. In this work, we

* Correspondence: nader.ghobadi@gmail.com

Physics Department, Faculty of Science, Malayer University, Malayer, Iran investigate the temperature dependence of dc conductivity of CdSe semiconducting nanoparticle films.

\section{Methods}

The deposition solution was prepared by a process similar to that used by Mane and Lokhande [6]. A volume of $400 \mathrm{ml}$ of $0.25 \mathrm{M}$ cadmium acetate (to provide $\mathrm{Cd}^{2+}$ ions) was taken in a glass beaker of $1,000 \mathrm{ml}$ capacity, under constant stirring, and then $25 \%$ ammonia was added to this solution slowly. At first, the solution become milky, and further addition of excess ammonia made the solution clear and transparent. Freshly prepared $0.25 \mathrm{M} \mathrm{Na}_{2} \mathrm{SeSo}_{3}$ (400 ml) [5] was added slowly to the solution. The CdSe films were grown on ordinary glass slides. Before deposition, the substrates were washed in detergent, rinsed in acetone, ultrasonically cleaned, and finally rinsed again with a mixture of double distilled water and methanol.

The glass substrates were vertically immersed into the deposition solution, and the bath solution was covered. In order to control the rate of films growth, the bath temperature was kept constant at room temperature. In order to control the $\mathrm{pH}$, ammonia is added to the solution, which contains $\mathrm{Cd}^{2+}$ ions. At the end of deposition process, all the deposited substrates were removed from the chemical bath at suitable intervals ( 4 to $24 \mathrm{~h}$ ) and then washed with deionized water and methanol to remove the loosely adhered CdSe nanoparticles on the 
film. The coating of one side of each substrate was removed by cotton swab moistened with dilute $\mathrm{HCl}$, and then the films were dried in the air and finally placed in the desiccators. The overall reaction is as follows [5]:

$$
\left[\mathrm{Cd}\left(\mathrm{NH}_{3}\right)_{4}\right]^{2+}+\mathrm{Se}^{2-} \rightarrow \mathrm{CdSe}+4 \mathrm{NH}_{3} .
$$

The films were structurally characterized by X-ray diffraction (XRD) using a Philip Analytical X-ray diffractometer in the $2 \theta$ geometry (Koninklijke Philips N.V., The Netherlands). The morphology of the films' surface was investigated by scanning electron microscopy. The dc electrical conductivity CdSe films was measured in a cryogenic Janis-CCS45 (Janis Research Company, LLC, Woburn, USA) system by a two-probe method in the temperature range of 300 to $450 \mathrm{~K}$, using Keithley 610C (Keithley Instruments Inc., Cleveland, USA) and TTi-196Gp (Thurlby Thandar Instruments Ltd., Cambridgeshire, England) electrometers for current and voltage measurements. For this purpose, silver paint electrodes were painted on the samples in a coplanar configuration.

\section{Results and discussion Optical absorption}

The absorption data were analyzed using the following well-known relation for near-edge optical absorption of semiconductors $[5,7]$,

$$
\alpha=A\left(h v-E_{\mathrm{g}}\right)^{n} / h v,
$$

where $\alpha$ is the absorption coefficient, $(h v)$ is the photon energy, $A$ is a constant, $E_{\mathrm{g}}$ is the optical gap, and $n$ is a constant equal to $1 / 2$ for direct gap semiconductors and 2 for indirect gap semiconductors [5]. To estimate the optical band gap, the plot of $(\alpha h v)^{2}$ versus $(h v)$ is shown in Figure 1. For as-deposited CdSe thin films, the best linear fit was obtained for $n=1 / 2$ with various deposition times.

Brus considered a model for the energy band gap with particle size that yields the following expression for the band gap, $E_{\mathrm{R}}$, of the quantum dot,

$$
E_{\mathrm{R}}=E_{\mathrm{g}} \hbar^{2} \pi^{2} / 2 R^{2}\left[1 / m_{\mathrm{e}}+1 / m_{\mathrm{h}}\right]-1.8 e^{2} / \varepsilon R,
$$

where $E_{\mathrm{g}}$ is the bulk band gap, and $R$ is the radius of the quantum dot. The third term arises due to the Coulomb attraction [8].

Dependence of the optical gap on the deposition time for the as-deposited thin films is shown in Figure 1. It is obvious that the optical gap decreases with increasing deposition time. This nanocrystalline films showed very strong confinement effects, as the reduction of grain size increases its energy band gap.

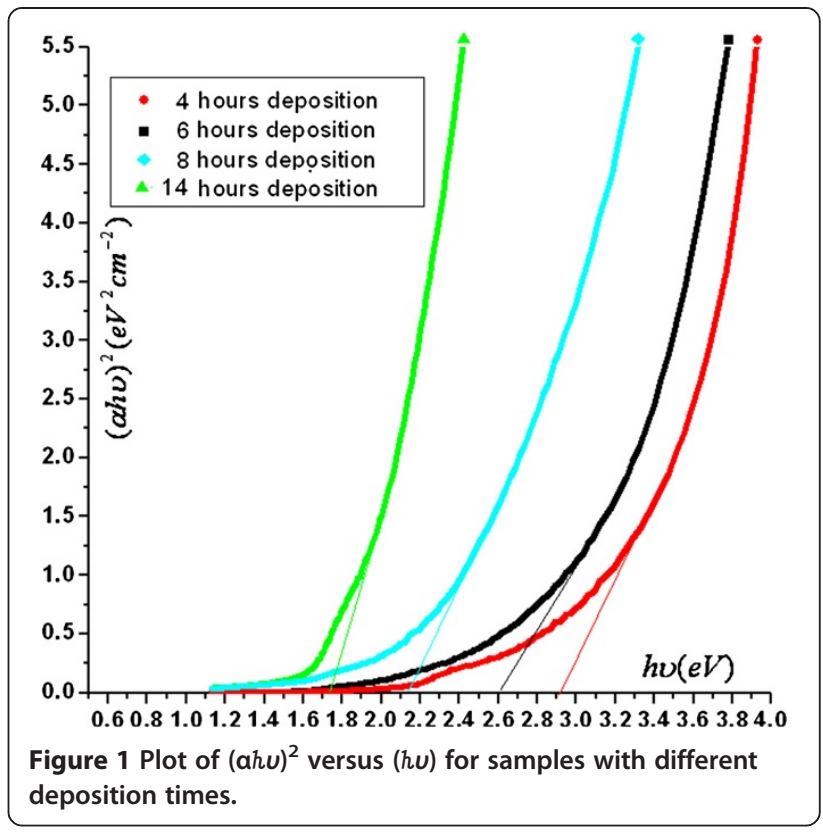

\section{Microstructural and XRD studies}

XRD patterns The representative X-ray diffraction patterns for some of CBD-CdSe films are shown in Figure 2; the summary of the obtained results are presented in Table 1. As $\mathrm{pH}$ and deposition time are increased, the intensity and the line width of the XRD patterns $\left(\beta_{\mathrm{hkl}}\right)$ get higher and smaller, respectively.

For the present samples, the X-ray patterns illustrate that the crystal structure of the CBD-CdSe is cubic [5]; for both as-deposited and samples with different $\mathrm{pH}$ values, no phase transition occurred.

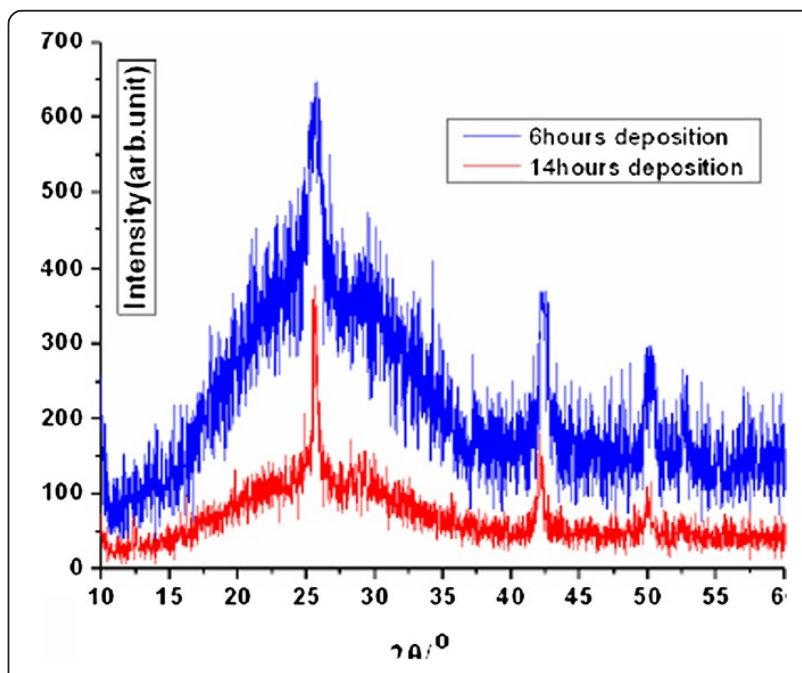

Figure 2 Representative XRD patterns of samples with 6- and 14-h deposition times. 
Table 1 Data from XRD patterns for CdSe nanoparticle films prepared at different PH and deposition times

\begin{tabular}{|c|c|c|c|c|c|}
\hline Film description & Number of peaks & d (Å) observed & $\beta_{\mathrm{hkl}}$ (radian) & $2 \theta /^{\circ}$ & (hkl) planes \\
\hline $\mathrm{pH}=11.25$ & 3 & 3.50091 & 1.15 & 25.68 & 111 \\
\hline \multirow[t]{2}{*}{ Deposition time $=6 \mathrm{~h}$} & & 2.14792 & 1.23 & 42.15 & \\
\hline & & 1.82922 & 0.62 & 49.94 & \\
\hline $\mathrm{pH}=11.6$ & 2 & 3.4689 & 0.80 & 25.66 & 111 \\
\hline Deposition time $=6 \mathrm{~h}$ & & 2.14362 & 0.96 & 42.12 & 220 \\
\hline $\mathrm{pH}=11.6$ & 3 & 3.49232 & 0.48 & 25.485 & 111 \\
\hline \multirow[t]{2}{*}{ Deposition time $=14 \mathrm{~h}$} & & 2.14265 & 0.40 & 42.140 & 220 \\
\hline & & 1.82473 & 0.64 & 49.940 & 311 \\
\hline $\mathrm{pH}=12.1$ & 3 & 3.47243 & 0.44 & 25.465 & 111 \\
\hline \multirow[t]{2}{*}{ Deposition time $=6 \mathrm{~h}$} & & 2.14162 & 0.36 & 42.110 & 220 \\
\hline & & 1.82375 & 0.62 & 49.940 & 311 \\
\hline
\end{tabular}

\section{Scanning electron microscopy}

Representative micrographs are shown in Figure 3; Figure 3 shows the scanning electron microscopy pictures of the 6- (a) and 14-h (b) deposition times at $\mathrm{pH}=11.6$ with magnifications $\times 30,000$. It can be observed that $\mathrm{CdSe}$ thin films cover the substrates. From the pictures, it is obviously seen that the film composed of small nanosized grains.

\section{Electrical conductivity}

In disordered materials such as amorphous semiconductor or some thin films, low temperature transport occurs between localized electronic states near the $E_{\mathrm{F}}$. This transport depends on the DOS, location of Fermi level and the temperature. Generally, at low temperatures (below about RT) and sometimes at temperatures above $\mathrm{RT}$, the electrical conduction is interpreted by VRH $[1,2]$; in this region, Mott's treatment of VRH leads to a temperature dependence for the electrical conduction of the form:

$$
\sigma=\sigma_{\mathrm{o}} \exp \left[-\left(T_{\mathrm{o}} / T\right)^{1 / 4}\right]
$$

where $T_{\mathrm{o}}$ and $\sigma_{\mathrm{o}}$ are parameters related to the localization of wave functions $\gamma$, density of states at (or near) the Fermi level $g\left(E_{\mathrm{F}}\right)$, optical phonon frequency $\nu_{\mathrm{o}}$, and the Boltzmann constant $K_{\mathrm{B}} ; T_{\mathrm{o}}$ is given by:

$$
T_{\mathrm{o}}=C_{\mathrm{o}} \gamma^{3} / K_{\mathrm{B}} g\left(E_{\mathrm{F}}\right)
$$

Where $C_{\mathrm{o}}$ is a constant, which has a value of approximately 16 (in the Mott's model [4], constant DOS) to about 310 (in the Godet's model [3], exponential behavior of DOS). Also, $1 / \gamma$ is the decay length of the electronic wave function which typically ranges from 3 to 30 $\AA$ [1]. As mentioned before this, VRH behavior of conduction is observed in a number of thin film materials

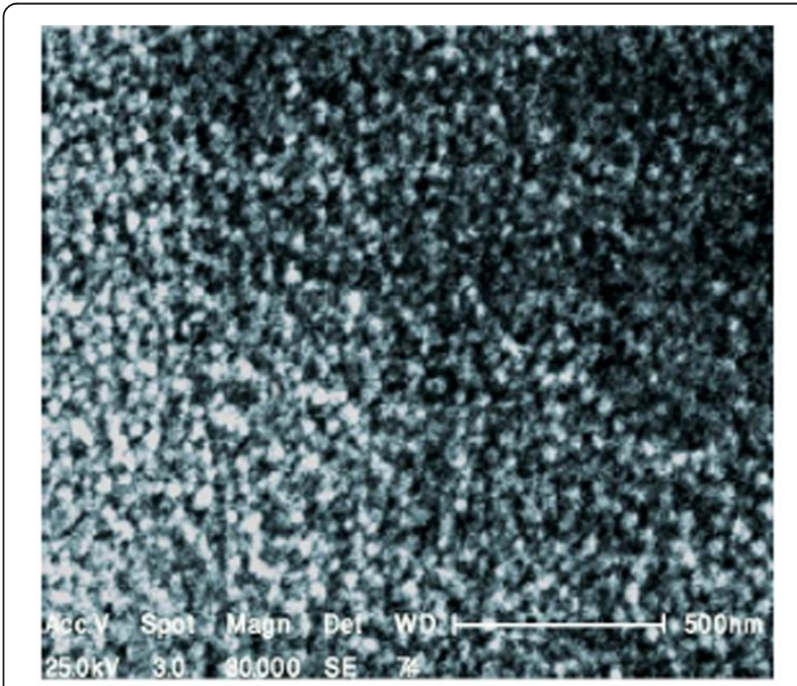

a: deposition time $=6 \mathrm{~h}$

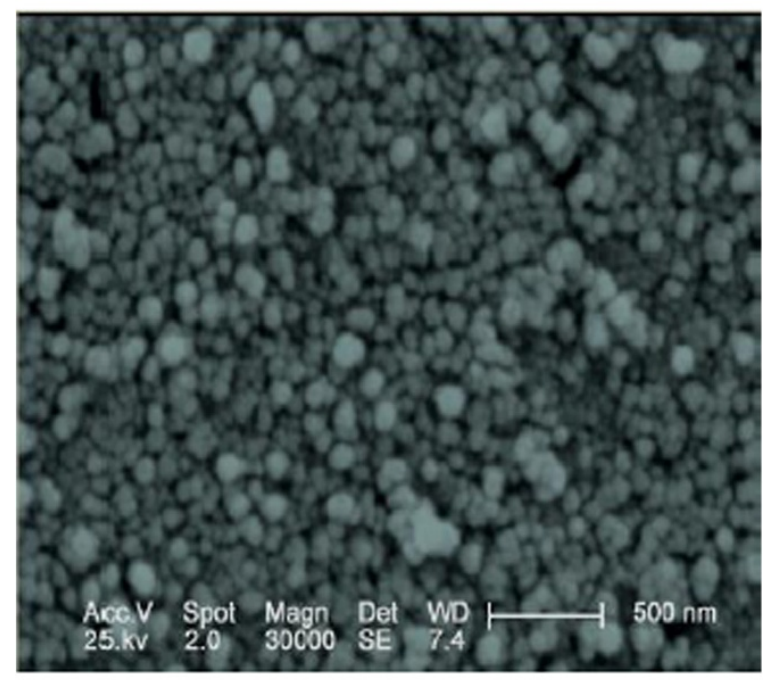

b: deposition time $=14 \mathrm{~h}$

Figure 3 Sample with 6-h deposition (a) and 14-h deposition time (b). 


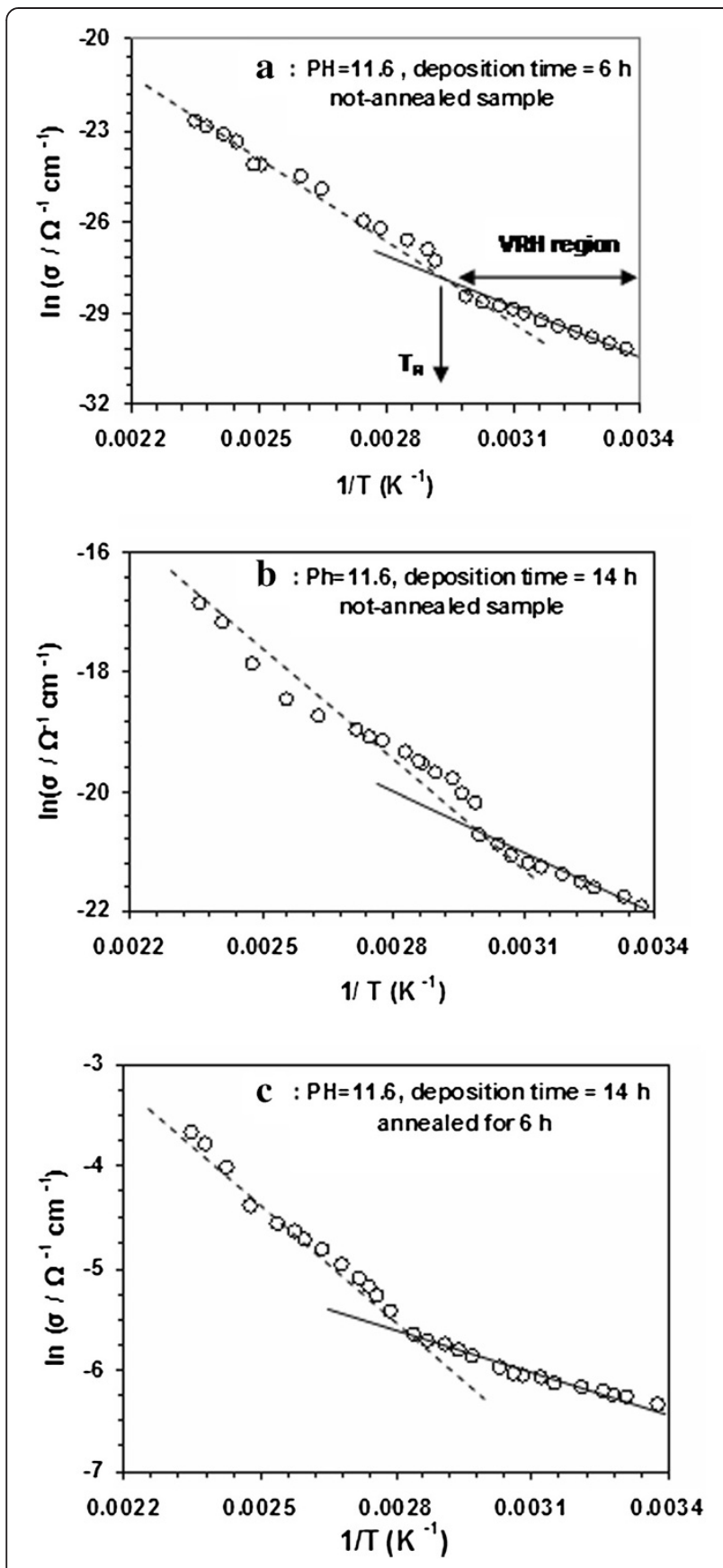

Figure 4 Temperature dependence of dc conductivity for CdSe nanoparticle films. $\mathrm{pH}=11.6$ for the best clarity; curves have been plotted separately. (a) $\mathrm{pH}=11.6$, deposition time $=6 \mathrm{~h}$, non-annealed sample. (b) $\mathrm{pH}=11.6$, deposition time $=14 \mathrm{~h}$, non-annealed sample. (c) $\mathrm{pH}=11.6$, deposition time $=14 \mathrm{~h}$, non-annealed sample.

and not only at low temperatures and constant DOS, as was the original Mott's prediction, but also at high temperatures in nanocrystalline [1] and disordered materials $[2,4]$. By considering the model proposed by Godet [3] for disordered materials and the similarity in conduction mechanism (VRH) of disordered and nanocrystalline semiconductors, we attempt to estimate the DOS of the CdSe nanoparticle films.

As discussed later, using Figures 4 and 5 and Equations 4 and 5, the DOS of CdSe are evaluated. The values of $\sigma_{\mathrm{o}}, T_{\mathrm{o}}, \mathrm{DOS}$, and localization parameter (LP) $g\left(E_{\mathrm{F}}\right) \gamma^{-3}$ obtained for CdSe nanoparticle films are summarized in Table 2 (values of $C_{\mathrm{o}}=16$ and 310 , and $1 / \gamma=$ $10 \AA$ have been used). It was observed that the DOS near the Fermi level increases with increase of grain size in films. It was observed that with increasing deposition time, activation energy is decreased, while the density of states is increased. We found that the density of states increased when annealing time is increased, while the activation energy decreased. These results show that the value of the density of states strongly depends on the annealing operation. We found that annealing times up to $6 \mathrm{~h}$ is very important; for the samples with lower annealing time variation of activation energy, density of states is less compared to the $6 \mathrm{~h}$ samples. Figure 4 shows the plot of $\ln \sigma$ versus $1 / T$ for the present samples. All of these curves show a slope change in characteristic temperature $\left(T_{\mathrm{R}}\right)$ of 330 to $340 \mathrm{~K}\left(T_{\mathrm{R}}\right.$ has been obtained from the intersection of solid and dashed lines in each curve of Figure 4). As is clear in Figure 4, there are two regions with different slopes in each curve, one above $T_{\mathrm{R}}$ and another below $T_{\mathrm{R}}$; the slope of $\ln \sigma-T^{-1}$ plot in the higher temperature region (called as $E_{\mathrm{a}} / K_{\mathrm{B}} T$, where $E_{\mathrm{a}}$ is the activation energy) can be obtained. Figure 5 shows the plot of $\ln \sigma-T^{-1 / 4}$ in the lower temperature region (VRH region) of Figure 4. In the lower temperature range (region of lower slope, VRH region), the parameters $T_{\mathrm{o}}, \sigma_{\mathrm{o}}$, and DOS have been obtained from the slope of the plot of $\ln \sigma$ versus $T^{-1 / 4}$ and its intercept at $T^{-1 / 4}=0$ by employing the least squares method. The linear correlation factors were between 0.9987 and 0.9993. All of the obtained results are listed in Table 2. Recent works on

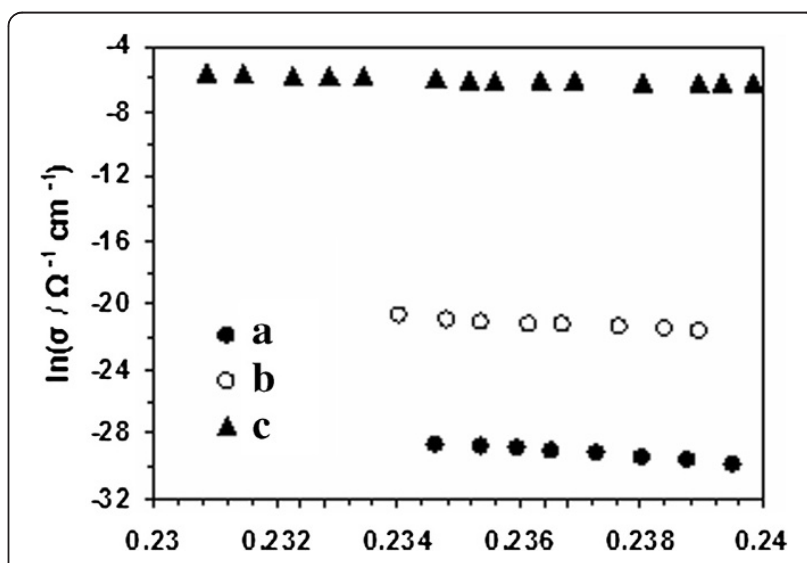

Figure 5 Plot of $1 \mathrm{n} \sigma$ versus $T^{1 / 4}$ (at VRH region, lower slope region in Figure 4) for $\mathrm{CdSe}$ nanoparticle films. $\mathrm{pH}=11.6$. (a), (b), and (c) are those mentioned in Table 2 and Figure 4. 
Table 2 Summary of the data obtained from electrical conduction CdSe nanoparticle films at $\mathbf{p H}=\mathbf{1 1 . 6}$

\begin{tabular}{|c|c|c|c|c|c|c|}
\hline Film description & $T_{\mathrm{o}}(\mathrm{K})$ & $\begin{array}{c}g\left(E_{\mathrm{F}}\right)\left(C_{\mathrm{o}}=16\right) \\
\left(\mathrm{eV}^{-1} \mathrm{~cm}^{-1}\right) \\
\text { Mott's model }\end{array}$ & $\begin{array}{c}g\left(E_{\mathrm{F}}\right)\left(C_{\mathrm{o}}=310\right) \\
\left(\mathrm{eV}^{-1} \mathrm{~cm}^{-1}\right) \\
\text { Godet's model }^{-1}\end{array}$ & $\begin{array}{l}\mathrm{LP}\left(C_{\mathrm{o}}=16\right)\left(\mathrm{eV}^{-1}\right) \\
\text { Mott's model }\end{array}$ & $\begin{array}{c}\mathrm{LP}\left(C_{\mathrm{o}}=310\right)\left(\mathrm{eV}^{-1}\right) \\
\text { Godet's model }\end{array}$ & $\begin{array}{c}E_{\mathrm{a}}(\mathrm{eV}) \text { at higher } \\
\text { temperature } \\
\text { region }\end{array}$ \\
\hline \multicolumn{7}{|l|}{ (a) } \\
\hline Non-annealed & $3.8 \times 10^{9}$ & $4.84 \times 10^{16}$ & $9.4 \times 10^{17}$ & $4.84 \times 10^{-5}$ & $4.84 \times 10^{-4}$ & 0.68 \\
\hline \multicolumn{7}{|l|}{ Deposition time $=6 \mathrm{~h}$} \\
\hline \multicolumn{7}{|l|}{ (b) } \\
\hline Non-annealed & $7.4 \times 10^{8}$ & $2.5 \times 10^{17}$ & $4.85 \times 10^{18}$ & $2.5 \times 10^{-4}$ & $2.5 \times 10^{-3}$ & 0.41 \\
\hline \multicolumn{7}{|l|}{ Deposition time $=14 \mathrm{~h}$} \\
\hline \multicolumn{7}{|l|}{ (c) } \\
\hline $6 \mathrm{~h}$ annealed & $1.37 \times 10^{7}$ & $1.37 \times 10^{19}$ & $2.65 \times 10^{20}$ & $1.3 \times 10^{-2}$ & $1.3 \times 10^{-1}$ & 0.32 \\
\hline Deposition time $=14 \mathrm{~h}$ & & & & & & \\
\hline
\end{tabular}

the electrical properties of semiconductor nanocrystalline have paid considerable attention toward the electrical conduction mechanism. In the nanocrystalline materials, due to small size of grains and large grain boundaries, the electronic states near the Fermi level are localized. When the states are localized, the conduction occurs by hopping of carriers between the occupied and unoccupied localized states which depends on the density of state and the position of the Fermi level.

In summary, as illustrated in Tables 1 and 2, the density of localized states near the Fermi level have been deduced to increase with the increase of grain size in the CdSe nanoparticle films, which justifies Godet's model. The gradual decrease in the activation energy (existence of different slopes in the plots of conductivity) with temperature suggests that the conduction is due to the hopping of the carriers among the localized states. We suggest that the samples made by this method have a convenient characteristic for the investigation of nanosensing properties, because the density of state and electrical conduction change by external subject.

\section{Conclusions}

In this work, the effects of deposition time and annealing on the density of states of CdSe nanoparticle films, prepared by chemical bath deposition, were investigated. It was observed that in the higher $\mathrm{pH}$, the grain size increased. In the nanocrystalline films, the charge carriers confined inside the nanoparticles, due to the reduction of grains size hence the electronic states close to the Fermi level, are localized. The gradual decrease in the activation energy with temperature suggests that the conduction is due to the hopping of the carriers among the localized states. The density of localized states near the Fermi level has been deduced to increase with increase of grain size in the CdSe nanoparticle films, in agreement with Godet's model. Our calculated localization parameters agreed with very small values of LP, where it corresponds to high temperature or low conduction. The localization parameters are due to confinement effect and confined charge carrier. For our system, modeling of ohmic conductivity for hopping within exponential distributions is useful in comparison with Mott's assumption of constant density of state near Fermi level. We suggest that the samples made by this method have a convenient characteristic for the investigation of nanosensing properties, because density of state and electrical conduction change by external subject.

\section{Competing interests}

The authors declare that they have no competing interests.

\section{Authors' contributions}

Both authors read and approved the final manuscript.

Received: 28 March 2013 Accepted: 3 July 2013

Published: 30 Jul 2013

\section{References}

1. Joshi RK, Sehgal H: Density of states near Fermi level in PbS nanoparticle films. Physica E. 23, 168 (2004)

2. Concari SB, Buitrago RH: Electronic transport mechanism in intrinsic and p-doped microcrystalline silicon thin films. J. Appl. Phys. 9(4):2417 (2003)

3. Godet C: Variable range hopping revisited: the case of an exponential distribution of localized states. J. Non-Cryst. Solids 333, 299-302 (2002)

4. Mott NF, Davis EA: Electron Processes in Non-Crystalline Materials. Oxford, Clarendon (1979)

5. Kale RB, Lokhande CD: Band gap shift, structural characterization and phase transformation of CdSe thin films from nanocrystalline cubic to nanorod hexagonal on air annealing. Semicond. Sci. Technol. 20, 1-9 (2005)

6. Mane RS, Lokhande CD: Chemical deposition method for metal chalcogenide thin films. Mater. Chem. Phys. 65, 1-31 (2000)

7. Tauc J, Menth A: States in the gap. J. Non-Cryst. Solids 569, 8-10 (1972)

8. Brus LE: Quantum crystallites and nonlinear optics. Appl. Phys. A 53, 465 (1991)

10.1186/2228-5326-3-47

Cite this article as: Ghobadi and Moradian: Strong localization of the charge carriers in CdSe nanostructural films. International Nano Letters 2013, 3:47 